\title{
Effects of solvent immiscibility on the phase behavior and microstructural length scales of a diblock copolymer in the presence of two solvents
}

\author{
Ching-I Huang* and Yu-Chieh Hsu \\ Institute of Polymer Science and Engineering, National Taiwan University, Taipei 106, Taiwan
}

(Received 23 June 2006; published 13 November 2006)

\begin{abstract}
We employ self-consistent mean-field theory to study the phase behavior and the microstructural sizes of $A B$ diblock copolymers in the presence of a neutral solvent $S_{1}$ and a slightly $B$-selective solvent $S_{2}$. In particular, the effects of copolymer volume fraction $\bar{\phi}_{C}$, the solvent ratio, and the immiscibility parameter between two solvents $\chi_{S_{1} S_{2}}$, are examined. We find that increasing $\chi_{S_{1} S_{2}}$ not only enlarges the ordered microphase region in the concentrated solutions, but also induces a less concentrated homogeneous solution to form an ordered structure and even undergo a macrophase separation. This is due to the fact that increasing $\chi_{S_{1} S_{2}}$ enhances the preferentially of $S_{1}$ for $A$ and $S_{2}$ for $B$ and, thereafter, the effective segregation between $A$ and $B$. Hence, we observe that the structural length results obtained by varying $\chi_{S_{1} S_{2}}$ resemble a consequence of varying the solvent selectivity in the diblock copolymer solutions when only one solvent is added. For example, when $\chi_{S_{1} S_{2}}$ is small, the domain spacing decreases with decreasing $\bar{\phi}_{C}$ while at larger values of $\chi_{S_{1} S_{2}}$, it first shows a decreasing trend and then an increasing behavior with decreasing $\bar{\phi}_{C}$.
\end{abstract}

DOI: 10.1103/PhysRevE.74.051802

PACS number(s): $61.25 . \mathrm{Hq}$

\section{INTRODUCTION}

Block copolymers, because of the immiscibility of the covalently bonded blocks, can form a rich variety of morphologies [1-5]. These self-assembled ordered structures typically range between $1-100 \mathrm{~nm}$ in size, and have valuable nanotechnological applications. One of the major methods in controlling the structural type as well as the length scale of these microstructures is by diluting a block copolymer with solvents. Many of the block copolymer systems with valuable technological applications contain block copolymers, oil, and water [4]. Therefore, it is important to study the phase behavior of a block copolymer in the presence of two solvents. A few experimental studies on poly(ethylene oxide- $b$-propylene oxide- $b$-ethylene oxide) (PEOPPO-PEO) triblock copolymers mixed with water and oil have been performed [6-12]. Due to the fact that water and oil are strongly immiscible, the swelling ability of both hydrophilic and hydrophobic groups in the block copolymers varies with the solvent ratio and solvent amount. As a result, various microstructures with different interfacial curvatures, such as lamellae, normal (oil-in-water) and reverse (waterin-oil) bicontinuous phase, normal and reverse cylinders, and normal and reverse spheres, form in the ternary mixtures of block copolymers, water, and oil. Indeed, phase behavior for a block copolymer in two solvents becomes very complicated as it involves many important parameters, such as the immiscibility between two solvents, solvent amount, solvent ratio, and the selectivity of two solvents for each block. To our knowledge, few of these factors have been fully analyzed theoretically, although there exist a few studies on the examination of phase behavior and the interfacial properties for a surfactant added into very immiscible oil and water by Monte Carlo simulations [13-16] and dissipative particle dy-

*Author to whom all correspondence should be addressed. FAX: 886-2-33665237. Email address: chingih@ntu.edu.tw namics (DPD) $[17,18]$. In this article, we employ selfconsistent mean-field (SCMF) theory to study both the phase behavior and the microstructural sizes of a diblock copolymer in two solvents. In particular, the effects of the incompatibility parameter between two solvents, the solvent ratio, and the copolymer volume fraction are examined.

It is well known that the SCMF theory has been proven a powerful technique for the study of the phase behavior in block copolymer systems. A systematic review of this theoretical development has been given by Shi [19]. By introducing the crystalline symmetry of the ordered phases and solving the SCMF equations in the reciprocal space, the resulting equilibrium phase diagram in terms of $\chi_{A B} N$ and the copolymer composition $f$ [where $\chi_{A B}$ is the Flory-Huggins interaction parameter between $A$ and $B, N$ is the degree of copolymerization, and $\left.f=N_{A} /\left(N_{A}+N_{B}\right)\right]$ for an $A B$ diblock copolymer melt has been obtained [20]. The composition $f$ largely determines the geometry of the microstructure, in which the shorter blocks form the minor domains. When a solvent $S$ is added to an $A B$ diblock copolymer, its selfassembling behaviors become more diverse, a result of the interplay of the effects of $N, f$, copolymer volume fraction $\phi$, and three independent interaction parameters, $\chi_{A B}, \chi_{A S}$, and $\chi_{B S}$. In the neutral case (i.e., $\chi_{A S}=\chi_{B S}$ ), provided the concentrated regime and the solvent quality is good, SCMF calculations have shown that the equilibrium solution phase maps are almost identical to the melt phase map when replaced with $\chi_{A B} N$ with $\phi \chi_{A B} N[21-24]$, the so-called "dilution approximation" [25]. To explain further, the order-order transitions (OOTs) and order-disorder transition (ODT) for concentrated solutions follow $\left(\phi \chi_{A B} N\right)_{\mathrm{ODT}, \mathrm{OOT}}=F(f)$, as given in the melts. Recently, experiments have shown that the dilution approximation is successful in predicting the OOTs [26-28] and microdomain spacings [26-30]. However, it fails to describe the ODT even for the concentrated block copolymer solutions due to the fluctuation effects [26-28,31]. When a selective solvent is added (i.e., $\chi_{A S} \neq \chi_{B S}$ ), the shape and the packing symmetry of the ordered structure is determined not 
only by the composition $f$ but also by the solvent selectivity. Banaszak and Whitmore [32] first employed the SCMF theory to investigate the lamellar phase in a selective solvent. They examined the lamellar microdomain spacing as a function of the copolymer volume fraction, degree of copolymerization, and solvent selectivity. Noolandi et al. [33] applied the SCMF theory to quantitatively determine the phase diagram of PEO-PPO-PEO triblock copolymers in aqueous solutions. Their calculated results are in significantly good agreement with experiments [34]. Huang and Lodge [24] then presented a numerical phase behavior for an $A B$ diblock copolymer in the presence of a solvent based on the SCMF theory. Although they only considered the classical phases, including lamellae $(L)$, hexagonally-packed cylinders $(C)$, and body-centered cubic array of spheres $\left(S^{\mathrm{BCC}}\right)$, they observed that both solvent selectivity and copolymer volume fraction strongly influence the morphology. In contrast to the neat diblock copolymer microstructures in which the shorter blocks form the minor domains, block copolymer solutions may form the "inverted" phases, where the longer blocks form the minor domains by varying solvent selectivity and copolymer volume fraction. Furthermore, the formed inverted spheres tend to pack from a body-centered cubic to face-centered cubic order upon increasing the solvent selectivity and/or solvent amount [35]. These theoretical results have been confirmed experimentally [26,27,36-40].

In this paper, we employ SCMF theory to examine the phase behavior and the microstructural sizes of an $A B$ diblock copolymer mixed with two solvents, $S_{1}$ and $S_{2}$. In particular, the effects of copolymer volume fraction $\bar{\phi}_{C}$, the solvent ratio, and the immiscibility parameter between two solvents $\chi_{S_{1} S_{2}}$ are examined. The model is formulated by a straightforward extension of our previous study of a block copolymer in the presence of one solvent in a canonical ensemble [24]. Using SCMF theory, we determine the equilibrium morphology and volume fraction profiles of each component. We then analyze how the distribution of each component affects the related microstructural domain length scales. As have been reported in the related experimental studies [6-12], the formed microstructure types are similar to those when only one solvent is added into a diblock copolymer. Hence, the possible phases needed to be considered here include $L$, gyroid $(G)$, perforated layers $(P L), C, S^{\mathrm{BCC}}$, and $S^{\mathrm{FCC}}$. For simplicity, we restrict consideration to the classical phases, such as $L, C, S^{\mathrm{BCC}}$, and $S^{\mathrm{FCC}}$, in order to construct the phase maps. In addition to the ordered microphases, one may expect that the systems with lower values of copolymer volume fraction $\bar{\phi}_{C}$ when $\chi_{S_{1} S_{2}}$ is large may macrophase separate into $S_{1^{-}}$and $S_{2}$-rich phases. In this case, it is better to adopt a grand canonical ensemble instead of our current SCMF model in a canonical ensemble approach. We thus use the Flory-Huggins thermodynamic analysis to locate the binodal coexistence curve between two disordered phases. Our SCMF results enable a more complete understanding of the rich and complex phase behavior that block copolymers exhibit when two solvents are added.

\section{THEORY}

\section{A. Self-consistent mean-field theory}

We use a canonical ensemble approach and consider a monodisperse $A B$ diblock copolymer in the presence of two solvents $S_{1}$ and $S_{2}$ with average volume fractions $\bar{\phi}_{C}, \bar{\phi}_{S_{1}}$, and $\bar{\phi}_{S_{2}}$, respectively. The degree of copolymerization is $N$ and $A$-monomer fraction in the copolymer is $f$. We assume that the system is incompressible both locally and globally, and each monomer type has the same statistical segment length $b$. The local interaction between each pair of monomers $I$ and $J$ is quantified by the Flory-Huggins interaction parameter $\chi_{I J}$. Each copolymer chain is parameterized by a variable $s$ that increases continuously from 0 to 1 along its length. We assume that the $A$-block starts from $s=0$ and terminates at $s=f$, the $A-B$ junction point.

In order to determine the concentration profiles as well as the free energy in the equilibrium state, we begin with solving the copolymer partial partition functions, $q_{C}(r, s)$ and $q_{C}^{+}(r, s)$, which are defined as the end-integrated distributions for a chain of $s N$ and (1-s) $N$ monomers, respectively, at $r$. The function $q_{C}(r, s)$ satisfies the following modified diffusion equation:

$$
\frac{\partial q_{C}}{\partial s}= \begin{cases}\frac{1}{6} N b^{2} \nabla^{2} q_{C}-\omega_{A} q_{C} & \text { if } s<f, \\ \frac{1}{6} N b^{2} \nabla^{2} q_{C}-\omega_{B} q_{C} & \text { if } s>f,\end{cases}
$$

and the initial condition is $q_{C}(r, 0)=1$. The equation for $q_{C}^{+}(r, s)$ is similar except that the right-hand side of Eq. (1) is multiplied by -1 , and the initial condition is $q_{C}^{+}(r, 1)=1$. In Eq. (1), $\omega_{A}(r)$ and $\omega_{B}(r)$ represent the fields acting on the $A$ and $B$ segments along the copolymer chains from $s=0$ to $f$ and from $s=f$ to 1 , respectively. The total partition function for a single copolymer chain $Q_{C}$ is found by integrating all possible configurations for the chains subject to these fields and thus equal to

$$
Q_{C}=\frac{1}{V} \int_{V} q_{C}(r, s=1) d^{3} r .
$$

For the solvent particles, the partition functions $Q_{S_{1}}$ and $Q_{S_{2}}$ subject to the fields $\omega_{S_{1}}(r)$ and $\omega_{S_{2}}(r)$, respectively, are simply equal to

$$
\begin{aligned}
& Q_{S_{1}}=\frac{1}{V} \int_{V} \exp \left[\frac{-\omega_{S_{1}}(r)}{N}\right] d^{3} r, \\
& Q_{S_{2}}=\frac{1}{V} \int_{V} \exp \left[\frac{-\omega_{S_{2}}(r)}{N}\right] d^{3} r,
\end{aligned}
$$

which indeed can be rewritten as

$$
Q_{S_{1}}=\frac{1}{V} \int_{V} q_{S_{1}}\left(r, s=\frac{1}{N}\right) d^{3} r,
$$




$$
Q_{S_{2}}=\frac{1}{V} \int_{V} q_{S_{2}}\left(r, s=\frac{1}{N}\right) d^{3} r,
$$

where $q_{S_{1}}(r, s)$ and $q_{S_{2}}(r, s)$ satisfy

$$
\begin{aligned}
& \frac{\partial q_{S_{1}}}{\partial s}=-\omega_{S_{1}} q_{S_{1}}, \\
& \frac{\partial q_{S_{2}}}{\partial s}=-\omega_{S_{2}} q_{S_{2}},
\end{aligned}
$$

with the initial conditions $q_{S_{1}}(r, s=0)=q_{S_{2}}(r, s=0)=1$.

Based on the minimization of free energy to attain thermodynamic equilibrium for equilibrium phases, the fields have to satisfy

$$
\begin{aligned}
& \omega_{A}(r)-\omega_{S_{2}}(r)= \chi_{A B} N \phi_{B}(r)+\chi_{A S_{1}} N \phi_{S_{1}}(r)+\chi_{A S_{2}} N \phi_{S_{2}}(r) \\
&-\chi_{A S_{2}} N \phi_{A}(r)-\chi_{B S_{2}} N \phi_{B}(r)-\chi_{S_{1} S_{2}} N \phi_{S_{1}}(r), \\
& \omega_{B}(r)-\omega_{S_{2}}(r)= \chi_{A B} N \phi_{A}(r)+\chi_{B S_{1}} N \phi_{S_{1}}(r)+\chi_{B S_{2}} N \phi_{S_{2}}(r) \\
&-\chi_{A S_{2}} N \phi_{A}(r)-\chi_{B S_{2}} N \phi_{B}(r)-\chi_{S_{1} S_{2}} N \phi_{S_{1}}(r), \\
& \omega_{S_{1}}(r)-\omega_{S_{2}}(r)= \chi_{A S_{1}} N \phi_{A}(r)+\chi_{B S_{1}} N \phi_{B}(r)+\chi_{S_{1} S_{2}} N \phi_{S_{2}}(r) \\
&-\chi_{A S_{2}} N \phi_{A}(r)-\chi_{B S_{2}} N \phi_{B}(r)-\chi_{S_{1} S_{2}} N \phi_{S_{1}}(r), \\
& \phi_{A}(r)+\phi_{B}(r)+\phi_{S_{1}}(r)+\phi_{S_{2}}(r)=1,
\end{aligned}
$$

where the volume fractions of $A, B, S_{1}$, and $S_{2}$, respectively, $\phi_{A}(r), \phi_{B}(r), \phi_{S_{1}}(r)$, and $\phi_{S_{2}}(r)$, have the following forms of

$$
\begin{gathered}
\phi_{A}(r)=\frac{\bar{\phi}_{C}}{Q_{C}} \int_{0}^{f} d s q_{C}(r, s) q_{C}^{+}(r, s), \\
\phi_{B}(r)=\frac{\bar{\phi}_{C}}{Q_{C}} \int_{f}^{1} d s q_{C}(r, s) q_{C}^{+}(r, s), \\
\phi_{S_{1}}(r)=\frac{\bar{\phi}_{S_{1}}}{Q_{S_{1}}} \exp \left[\frac{-\omega_{S_{1}}(r)}{N}\right]=\frac{\bar{\phi}_{S_{1}}}{Q_{S_{1}}} q_{S_{1}}\left(r, s=\frac{1}{N}\right), \\
\phi_{S_{2}}(r)=\frac{\bar{\phi}_{S_{2}}}{Q_{S_{2}}} \exp \left[\frac{-\omega_{S_{2}}(r)}{N}\right]=\frac{\bar{\phi}_{S_{2}}}{Q_{S_{2}}} q_{S_{2}}\left(r, s=\frac{1}{N}\right) .
\end{gathered}
$$

Once the above volume fractions are determined and the self-consistent equations for the fields are satisfied, the free energy per molecule $F$ is given by

$$
\begin{aligned}
\frac{F}{k_{B} T}= & -\bar{\phi}_{C} \ln \left[\frac{Q_{C}}{\bar{\phi}_{C}}\right]-\bar{\phi}_{S_{1}} N \ln \left[\frac{Q_{S_{1}}}{\bar{\phi}_{S_{1}}}\right]-\bar{\phi}_{S_{2}} N \ln \left[\frac{Q_{S_{2}}}{\bar{\phi}_{S_{2}}}\right] \\
& -\frac{1}{V} \int_{V}\left[\omega_{A}(r) \phi_{A}(r)+\omega_{B}(r) \phi_{B}(r)+\omega_{S_{1}}(r) \phi_{S_{1}}(r)\right. \\
& \left.+\omega_{S_{2}}(r) \phi_{S_{2}}(r)\right] d^{3} r
\end{aligned}
$$

$$
\begin{aligned}
& +\frac{N}{V} \int_{V}\left[\chi_{A B} \phi_{A}(r) \phi_{B}(r)+\chi_{A S_{1}} \phi_{A}(r) \phi_{S_{1}}(r)\right. \\
& \left.+\chi_{A S_{2}} \phi_{A}(r) \phi_{S_{2}}(r)\right] d^{3} r+\frac{N}{V} \int_{V}\left[\chi_{B S_{1}} \phi_{B}(r) \phi_{S_{1}}(r)\right. \\
& \left.+\chi_{B S_{2}} \phi_{B}(r) \phi_{S_{2}}(r)+\chi_{S_{1} S_{2}} \phi_{S_{1}}(r) \phi_{S_{2}}(r)\right] d^{3} r
\end{aligned}
$$

For a disordered state, $\phi_{I}(r)=\bar{\phi}_{I}$ and the fields $\omega_{I}$ are constants, $I=A, B, S_{1}, S_{2}$. The partition functions are simply $Q_{C}=\exp \left(-f \omega_{A}-(1-f) \omega_{B}\right), Q_{S_{1}}=\exp \left(-\omega_{S_{1}} / N\right)$, and $Q_{S_{2}}=\exp \left(-\omega_{S_{2}} / N\right)$. As a result, Eq. (8) is reduced to the Flory-Huggins mean-field free energy functional per molecule in the disordered state, i.e.,

$$
\begin{aligned}
\frac{F}{k_{B} T_{\text {disorder }}}= & \bar{\phi}_{C} \ln \bar{\phi}_{C}+\bar{\phi}_{S_{1}} N \ln \bar{\phi}_{S_{1}}+\bar{\phi}_{S_{2}} N \ln \bar{\phi}_{S_{2}} \\
& +f(1-f) \chi_{A B} N \bar{\phi}_{C}^{2}+f \chi_{A S_{1}} N \bar{\phi}_{C} \bar{\phi}_{S_{1}} \\
& +(1-f) \chi_{B S_{1}} N \bar{\phi}_{C} \bar{\phi}_{S_{1}}+f \chi_{A S_{2}} N \bar{\phi}_{C} \bar{\phi}_{S_{2}} \\
& +(1-f) \chi_{B S_{2}} N \bar{\phi}_{C} \bar{\phi}_{S_{2}}+\chi_{S_{1} S_{2}} N \bar{\phi}_{S_{1}} \bar{\phi}_{S_{2}} .
\end{aligned}
$$

For a periodic ordered phase, the free energy has to be minimized with respect to the lattice spacing $L$; to determine the most stable phase one has to compare free energies of possible phases. For simplicity, we restrict consideration to the classical phases, such as $L, C, S^{\mathrm{BCC}}, S^{\mathrm{FCC}}$. Other possible ordered phases such as $G$ and $P L$ are not examined here.

As the block copolymer morphologies are periodic, it is most efficient to perform the SCMF calculations using the Fourier-space algorithm. That is, any given function, $g(r)$, is expressed in terms of the corresponding amplitudes, $g_{j}$, with respect to a series of orthonormal basis function $f_{j}(r), g(r)$ $=\Sigma_{j} g_{j} f_{j}(r)$. The basis functions reflect the symmetry of the ordered phase being considered. The eigenfunctions of the Laplacian operator are selected as the basis functions

$$
\nabla^{2} f_{j}(r)=-\lambda_{j} L^{-2} f_{j}(r)
$$

where $L$ is the lattice spacing for the ordered phase. The basis functions are ordered starting with $f_{1}(r)=1$ such that $\lambda_{j}$ is an increasing series. For lamellae $f_{j}(r)=2^{1 / 2} \cos (2 \pi(j-1) x / L), j \geqslant 2$, where $x$ is the coordinate orthogonal to the lamellae. Basis functions for the phases with other space-group symmetries can be found in Ref. [41]. Note that the number of basis functions varies much with the ordered phase and the segregation degree. In any case, sufficient number of basis functions has to be included in our computations in order to assure that the results reach the equilibrium values.

When the amplitudes corresponding to the basis functions are utilized, Eqs. (1), (5a), and (5b), for solving $q_{C}(r, s)$, $q_{S_{1}}(r, s)$, and $q_{S_{2}}(r, s)$, become 


$$
\begin{gathered}
\frac{\partial q_{C, i}}{\partial s}=\left\{\begin{array}{l}
\sum_{j} A_{i j} q_{C, j} \text { if } s<f, \\
\sum_{j} B_{i j} q_{C, j} \quad \text { if } s>f,
\end{array} \quad i=1,2,3, \ldots,\right. \\
\quad \frac{\partial q_{S_{1}, i}}{\partial s}=\sum_{j} C_{i j} q_{S_{1}, j}, \quad i=1,2,3, \ldots, \\
\frac{\partial q_{S_{2}, i}}{\partial s}=\sum_{j} D_{i j} q_{S_{2}, j}, \quad i=1,2,3, \ldots .
\end{gathered}
$$

The equation for $q_{C, i}{ }^{+}$is similar except that the right-hand side of Eq. (11a) is multiplied by -1 . The initial conditions are $q_{C, i}(s=0)=\delta_{i 1}, \quad q_{C, i}{ }^{+}(s=1)=\delta_{i 1}, \quad q_{S_{1}, i}(s=0)=\delta_{i 1}, \quad$ and $q_{S_{2}, i}(s=0)=\delta_{i 1}$. The matrices $A_{i j}, B_{i j}, C_{i j}$, and $D_{i j}$ are given by

$$
\begin{gathered}
A_{i j}=-\frac{N b^{2}}{6 L^{2}} \lambda_{i} \delta_{i j}-\sum_{k} \omega_{A, k} \Gamma_{i j k}, \\
B_{i j}=-\frac{N b^{2}}{6 L^{2}} \lambda_{i} \delta_{i j}-\sum_{k} \omega_{B, k} \Gamma_{i j k}, \\
C_{i j}=-\sum_{k} \omega_{S_{1}, k} \Gamma_{i j k}, \\
D_{i j}=-\sum_{k} \omega_{S_{2}, k} \Gamma_{i j k},
\end{gathered}
$$

where $\Gamma_{i j k}=V^{-1} \int f_{i}(r) f_{j}(r) f_{k}(r) d^{3} r . \omega_{A, k}, \omega_{B, k}, \omega_{S_{1}, k}$, and $\omega_{S_{2}, k}$ are the corresponding amplitudes with respect to the $k$ th basis function for fields $\omega_{A}, \omega_{B}, \omega_{S_{1}}$, and $\omega_{S_{2}}$, respectively. From Eq. (6) it is straightforward that

$$
\begin{gathered}
\omega_{A, i}-\omega_{S_{2}, i}=\chi_{A B} N \phi_{B, i}+\chi_{A S_{1}} N \phi_{S_{1}, i}+\chi_{A S_{2}} N \phi_{S_{2}, i}-\chi_{A S_{2}} N \phi_{A, i} \\
-\chi_{B S_{2}} N \phi_{B, i}-\chi_{S_{1} S_{2}} N \phi_{S_{1}, i}, \\
\omega_{B, i}-\omega_{S_{2}, i}=\chi_{A B} N \phi_{A, i}+\chi_{B S_{1}} N \phi_{S_{1}, i}+\chi_{B S_{2}} N \phi_{S_{2}, i}-\chi_{A S_{2}} N \phi_{A, i} \\
-\chi_{B S_{2}} N \phi_{B, i}-\chi_{S_{1} S_{2}} N \phi_{S_{1}, i}, \\
\omega_{S_{1}, i}-\omega_{S_{2}, i}=\chi_{A S_{1}} N \phi_{A, i}+\chi_{B S_{1}} N \phi_{B, i}+\chi_{S_{1} S_{2}} N \phi_{S_{2}, i} \\
-\chi_{A S_{2}} N \phi_{A, i}-\chi_{B S_{2}} N \phi_{B, i}-\chi_{S_{1} S_{2}} N \phi_{S_{1}, i}, \\
\phi_{A, i}+\phi_{B, i}+\phi_{S_{1}, i}+\phi_{S_{2}, i}=\delta_{i 1},
\end{gathered}
$$

where the amplitudes of the volume fractions of $A, B, S_{1}$, and $S_{2}$, respectively, $\phi_{A, i}, \phi_{B, i}, \phi_{S_{1}, i}$, and $\phi_{S_{2}, i}$, are determined by

$$
\begin{aligned}
& \phi_{A, i}=\frac{\bar{\phi}_{C}}{q_{C, 1}(1)} \sum_{j, k} \Gamma_{i j k} \int_{0}^{f} d s q_{C, j}(s) q_{C, k}^{+}(s), \\
& \phi_{B, i}=\frac{\bar{\phi}_{C}}{q_{C, 1}(1)} \sum_{j, k} \Gamma_{i j k} \int_{f}^{1} d s q_{C, j}(s) q_{C, k}^{+}(s),
\end{aligned}
$$

$$
\phi_{S_{1}, i}=\frac{\bar{\phi}_{S_{1}}}{q_{S_{1}, 1}\left(\frac{1}{N}\right)} q_{S_{1}, i}\left(\frac{1}{N}\right)
$$

$$
\phi_{S_{2}, i}=\frac{\bar{\phi}_{S_{2}}}{q_{S_{2}, 1}\left(\frac{1}{N}\right)} q_{S_{2}, i}\left(\frac{1}{N}\right)
$$

and the free energy per molecule $F$ in Eq. (8) is expressed as

$$
\begin{aligned}
\frac{F}{k_{B} T}= & -\bar{\phi}_{C} \ln \left[\frac{q_{C, 1}(1)}{\bar{\phi}_{C}}\right]-\bar{\phi}_{S_{1}} N \ln \left[\frac{q_{S_{1}, 1}(1 / N)}{\bar{\phi}_{S_{1}}}\right] \\
& -\bar{\phi}_{S_{2}} N \ln \left[\frac{q_{S_{2}, 1}(1 / N)}{\bar{\phi}_{S_{2}}}\right] \\
& -\sum_{i}\left(\omega_{A, i} \phi_{A, i}+\omega_{B, i} \phi_{B, i}+\omega_{S_{1}, i} \phi_{S_{1}, i}+\omega_{S_{2}, i} \phi_{S_{2}, i}\right) \\
& +\sum_{i}\left(\chi_{A B} N \phi_{A, i} \phi_{B, i}+\chi_{A S_{1}} N \phi_{A, i} \phi_{S_{1}, i}\right. \\
& \left.+\chi_{A S_{2}} N \phi_{A, i} \phi_{S_{2}, i}\right)+\sum_{i}\left(\chi_{B S_{1}} N \phi_{B, i} \phi_{S_{1}, i}\right. \\
& \left.+\chi_{B S_{2}} N \phi_{B, i} \phi_{S_{2}, i}+\chi_{S_{1} S_{2}} N \phi_{S_{1}, i} \phi_{S_{2}, i}\right)
\end{aligned}
$$

In order to examine the effects of the solvent immiscibility on the structural length scales, as well as the distribution of each component within the ordered structures, we choose the particular parameters in which the lamellar phase is the most stable one. Later we will demonstrate that these lamellar length results when two solvents are added can be qualitatively extended by those results of a diblock copolymer in the presence of one solvent. Based on the fact that the effects of adding one solvent on the length scales within each ordered phase are qualitatively consistent [35], we believe similar results in the lamellar phase also hold true qualitatively in other structures when a second solvent is added. Once the lattice spacing $L$ is obtained, we determine the width of the $A$-rich regions $L_{A}$, the interfacial width $w$, and the width of the $B$-rich regions $L_{B}$, as follows. For example, when a neutral solvent $S_{1}\left(\chi_{A S_{1}}=\chi_{B S_{1}}=0.4\right)$ and a $B$-selective solvent $S_{2}\left(\chi_{A S_{1}}=0.6, \chi_{B S_{1}}=0.4\right)$ are added to a block copolymer with $f=0.5, N=200, \phi=0.5$, and $\chi_{A B} N=25, L$ is the most stable phase. Figure 1 shows the typical volume fraction profiles $\phi_{A}, \phi_{B}, \phi_{S_{1}}$, and $\phi_{S_{2}}$, from which the inflection points with respect to $\phi_{\mathrm{A}}$ and $\phi_{B}$; i.e., $d^{2} \phi_{\mathrm{I}} / d x^{2}=0(I=A, B)$ are determined. Note that these inflection points are identical. The $A$-rich domain width $L_{A}$ is determined as the distance between two intersection points, which are obtained by plotting the tangent line to $\phi_{A}$ at the point when $\phi_{A}$ is a maximum with those at the inflection points, as shown in Fig. 1. Similar procedures are performed to determine the $B$-rich domain width $L_{B}$. The interfacial width $w$ is thus equal to $L-L_{A}-L_{B}$. 


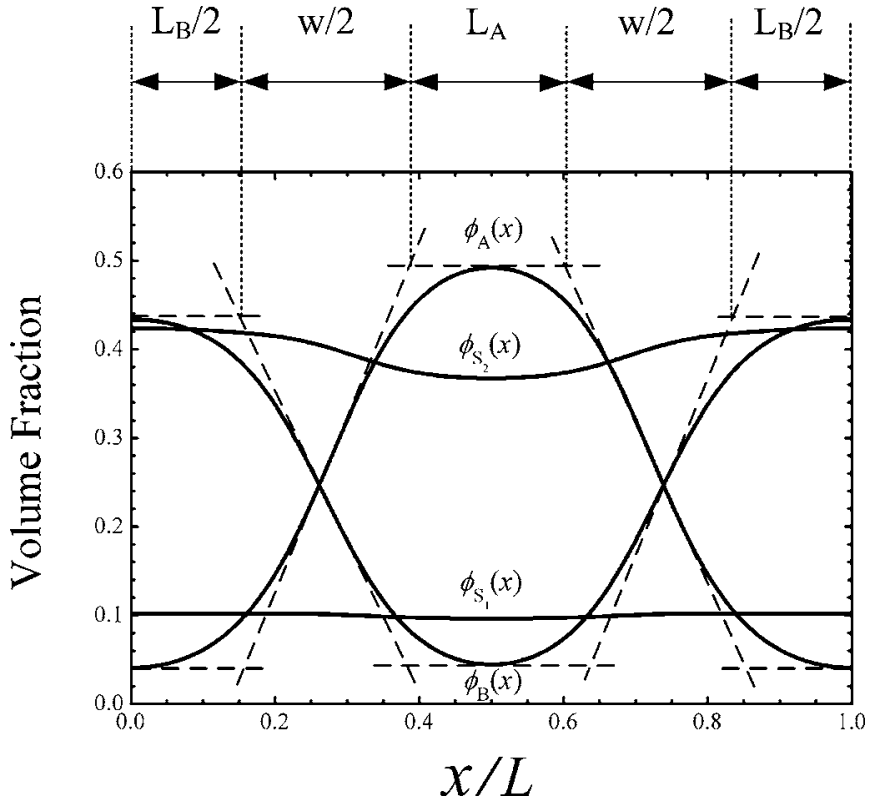

FIG. 1. Volume fraction profiles of $\phi_{A}, \phi_{B}, \phi_{S_{1}}$ and $\phi_{S_{2}}$ for a system in the lamellar phase with $f=0.5, N=200, \chi_{A B} N=25, \bar{\phi}_{C}$ $=0.5, \bar{\phi}_{S_{1}} / \bar{\phi}_{S_{2}}=2 / 8, \chi_{A S_{1}}=\chi_{B S_{1}}=0.4, \chi_{A S_{2}}=0.6$, and $\chi_{B S_{2}}=0.4$.

\section{B. Binodal coexistence curves between disordered phases}

For a ternary system, the thermodynamic analysis of determining the phase equilibrium has been described in detail elsewhere [42]. Here we briefly give the resulting equations for an $A B$ diblock copolymer in the presence of two solvents $S_{1}$ and $S_{2}$. To determine the coexistence between disordered phases $(\alpha, \beta, \ldots)$, one has to equate the chemical potential of each component $C(=A B), S_{1}$ and $S_{2}$, in the possible coexisting phases including the mass conservation laws, i.e.,

$$
\begin{gathered}
\mu_{I}^{(\alpha)}=\mu_{I}^{(\beta)}, \quad I=C, S_{1}, S_{2}, \\
\bar{\phi}_{I}=\nu^{\alpha} \bar{\phi}_{I}^{\alpha}+\nu^{\beta} \bar{\phi}_{I}^{\beta}, \quad I=C, S_{1}, S_{2},
\end{gathered}
$$

where $\nu^{m}$ is the volume fraction of phase $m$ and $\bar{\phi}_{I}^{m}$ is the mean volume fraction of component $I$ in the $m$ phase. The chemical potential of component $I$ is defined as the change of the total free energy $\Delta F$ 。 with respect to the number of molecules of component $I, n_{I}$,

$$
\begin{gathered}
\mu_{I}=\frac{\partial \Delta F_{\circ}}{\partial n_{I}}, \\
\Delta F_{\circ}=F \times\left(n_{C}+\frac{n_{S_{1}}}{N}+\frac{n_{S_{2}}}{N}\right),
\end{gathered}
$$

where $F$ is the Flory-Huggins mean-field free energy functional per copolymer molecule and has been given in Eq. (9). With a series of mathematical operations [42], $\mu_{I}, I=C, S_{1}$, $S_{2}$, has the form of
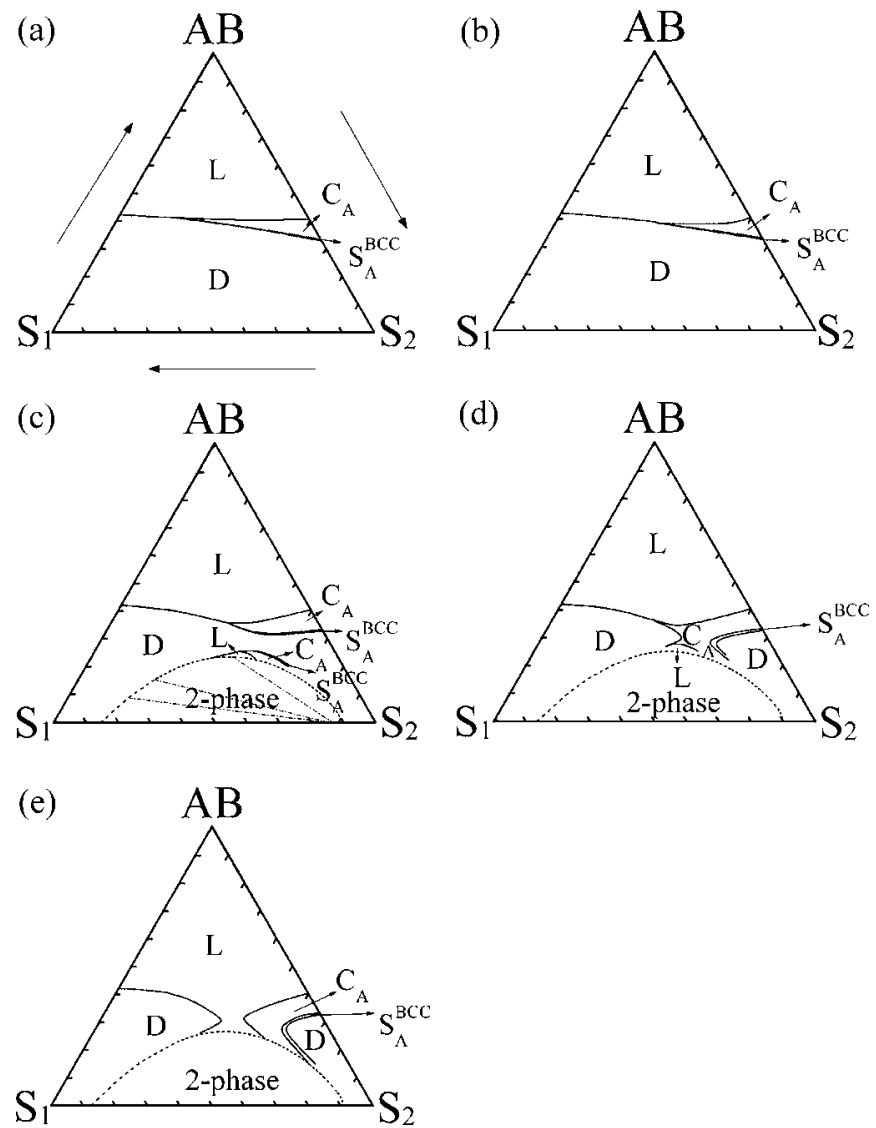

FIG. 2. Phase diagrams of an $A B$ diblock copolymer in the presence of two solvents $S_{1}$ and $S_{2}$ with $f=0.5, N=200 \chi_{A B} N=25$, $\chi_{A S_{1}}=\chi_{B S_{1}}=0.4, \chi_{A S_{2}}=0.6, \chi_{B S_{2}}=0.4$, and $\chi_{S_{1} S_{2}}=$ (a) 0.0 , (b) 2.0, (c) 2.5 , (d) 2.55 , and (e) 2.6, respectively. The (----) and (-.-.) lines correspond to the 2D coexistence curves and the tie-lines, respectively.

$$
\begin{aligned}
& \mu_{C}=k_{B} T\left\{\ln \phi_{C}+(1-N)\left(1-\phi_{C}\right)\right. \\
& +N\left[f \chi_{A S_{1}}+(1-f) \chi_{B S_{1}}\right] \phi_{S_{1}}\left(1-\phi_{C}\right) \\
& +N\left[f \chi_{A S_{2}}+(1-f) \chi_{B S_{2}}\right] \phi_{S_{2}}\left(1-\phi_{C}\right) \\
& \left.+N f(1-f) \chi_{A B} \phi_{C}\left(2-\phi_{C}\right)-N \chi_{S_{1} S_{2}} \phi_{S_{1}} \phi_{S_{2}}\right\} \text {, } \\
& \mu_{S_{1}}=k_{B} T\left\{\ln \phi_{S_{1}}+\phi_{C}\left(1-\frac{1}{N}\right)\right. \\
& +\left[f \chi_{A S_{1}}+(1-f) \chi_{B S_{1}}\right] \phi_{C}\left(1-\phi_{S_{1}}\right)+\chi_{S_{1} S_{2}} \phi_{S_{2}}\left(1-\phi_{S_{1}}\right) \\
& \left.-\left[f \chi_{A S_{2}}+(1-f) \chi_{B S_{2}}\right] \phi_{C} \phi_{S_{2}}-f(1-f) \chi_{A B} \phi_{C}^{2}\right\} \text {, } \\
& \mu_{S_{2}}=k_{B} T\left\{\ln \phi_{S_{2}}+\phi_{C}\left(1-\frac{1}{N}\right)\right. \\
& +\left[f \chi_{A S_{2}}+(1-f) \chi_{B S_{2}}\right] \phi_{C}\left(1-\phi_{S_{2}}\right)+\chi_{S_{1} S_{2}} \phi_{S_{1}}\left(1-\phi_{S_{2}}\right) \\
& \left.-\left[f \chi_{A S_{1}}+(1-f) \chi_{B S_{1}}\right] \phi_{C} \phi_{S_{1}}-f(1-f) \chi_{A B} \phi_{C}^{2}\right\} \text {. }
\end{aligned}
$$



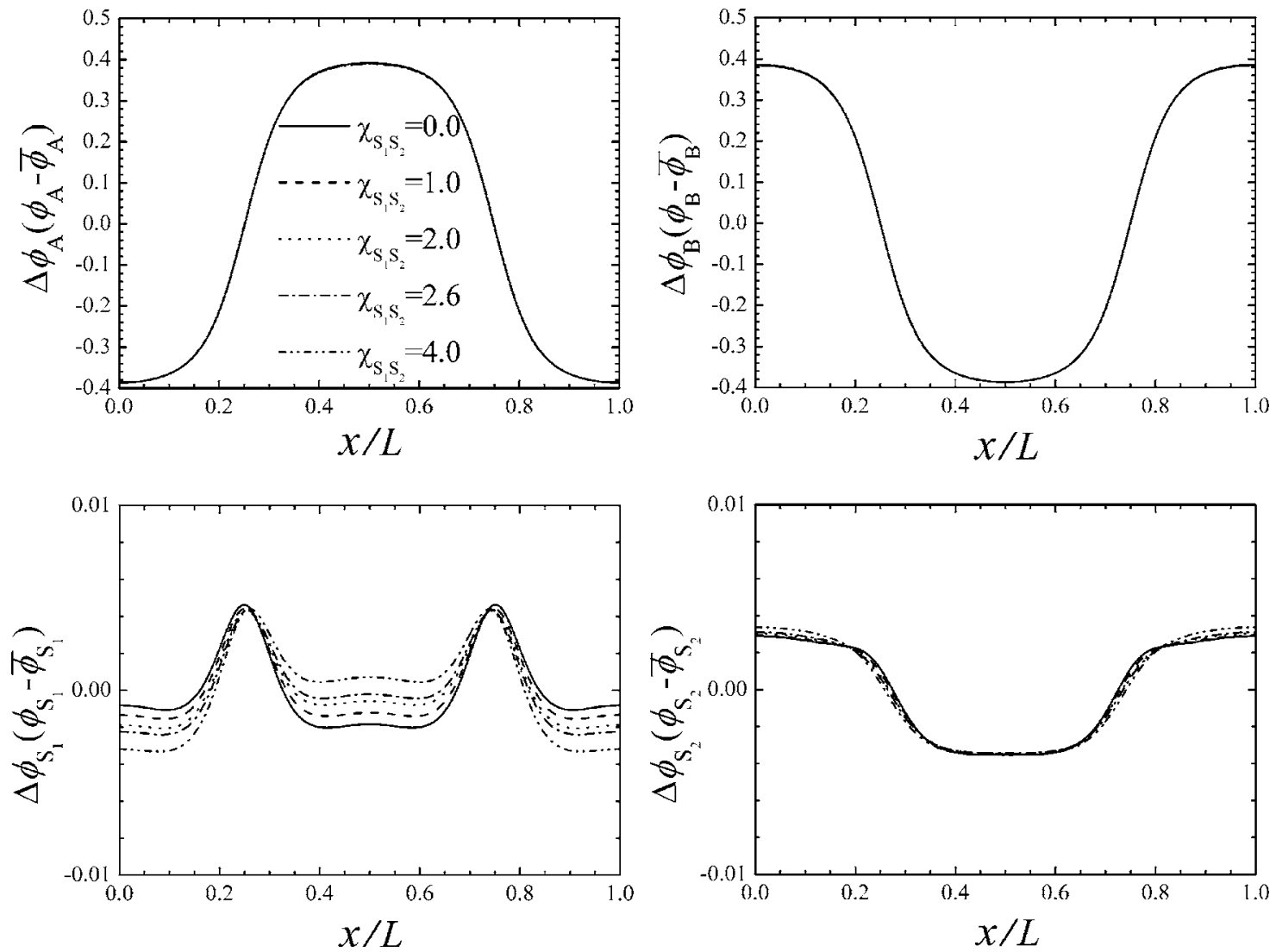

FIG. 3. Deviation of volume fraction profiles of component $A, B, S_{1}$, and $S_{2}$ within the lamellar phase for $f=0.5, N=200, \chi_{A B} N=25$, $\bar{\phi}_{C}=0.8, \bar{\phi}_{S_{1}} / \bar{\phi}_{S_{2}}=8 / 2, \chi_{A S_{1}}=\chi_{B S_{1}}=0.4, \chi_{A S_{2}}=0.6$, and $\chi_{B S_{2}}=0.4$ at various values of $\chi_{S_{1} S_{2}}$.

Note that the system is always assumed to be incompressible, i.e., $\phi_{C}+\phi_{S_{1}}+\phi_{S_{2}}=1$.

\section{RESULTS AND DISCUSSION}

To examine how the addition of two immiscible solvents affects the phase behavior and the microstructural domain length scales of block copolymers, we choose $f=0.5, N$ $=200$, and $\chi_{A B}=0.125$. As a result, the block copolymer melt is a lamellar phase at $\chi_{A B} N=25$. The solvent $S_{1}$ is a neutral solvent, and $S_{2}$ is a $B$-selective solvent. In particular, $\chi_{A S_{1}}$ $=\chi_{B S_{1}}=0.4, \chi_{A S_{2}}=0.6$, and $\chi_{B S_{2}}=0.4$.

Figure 2 presents a series of corresponding phase diagrams, which are obtained by SCMF calculations and shown in $A B-S_{1}-S_{2}$ composition triangles, at various values of the immiscibility parameter between two solvents $\chi_{S_{1} S_{2}}$. The Ith corner in the triangle represents a system composed of $100 \%$ component $I$. We first briefly discuss the effects of the addition of only one solvent $\left(S_{1}\right.$ or $\left.S_{2}\right)$ on the lamellar phase of diblock copolymers. When a neutral and good solvent $S_{1}$ is added to a symmetric lamellar diblock copolymer, in addition to the slight solvent accumulation behavior at the interfaces, it is expected to distribute equally in the $A$ - and $B$-rich domains. As a result, the addition of more $S_{1}$ to block copolymers reduces the effective segregation between $A$ and $B$, and a transition from $L$ to disordered phase $(D)$ occurs at $\bar{\phi}_{C, \mathrm{ODT}}=0.42$, according to the "dilution approximation." In the $B$-selective solvent $S_{2}$, due to the fact that $S_{2}$ prefers the $\mathrm{B}$ block and thus acts in a manner that corresponds qualitatively to reducing the $A$ composition $f$, a sequence $L \rightarrow$ hexagonally-packed $A$-formed cylinders $\left(C_{A}\right) \rightarrow$ bodycentered cubic array of $A$-formed spheres $\left(S_{A}^{\mathrm{BCC}}\right) \rightarrow D$ is observed as $\bar{\phi}_{C}$ decreases. The values of $\bar{\phi}_{C}$ at boundaries between $L / C_{A}, C_{A} / S_{A}^{\mathrm{BCC}}$, and $S_{A}^{\mathrm{BCC}} / D$, are equal to 0.403 , 0.329 , and 0.326 , respectively. Note that since $S_{2}$ is only slightly selective for $B$, it is more stable for the spheres formed by $A$ blocks at lower values of $\bar{\phi}_{C}$ packed in a bcc array than fcc, which is in a qualitatively good agreement with experimental results $[38,39]$.

When both ideally miscible solvents $S_{1}$ and $S_{2}\left(\chi_{S_{1} S_{2}}=0\right)$ are added, we observe that the resulting equilibrium phase behavior [shown in Fig. 2(a)] resembles a consequence of the effects of a selective solvent $S$, in which the effective interaction parameters between $A / S$ and $B / S$ are equal to $\bar{\phi}_{S_{1}} /\left(\bar{\phi}_{S_{1}}+\bar{\phi}_{S_{2}}\right) \chi_{A S_{1}}+\bar{\phi}_{S_{2}} /\left(\bar{\phi}_{S_{1}}+\bar{\phi}_{S_{2}}\right) \chi_{A S_{2}}$ and $\bar{\phi}_{S_{1}} /\left(\bar{\phi}_{S_{1}}\right.$ $\left.+\bar{\phi}_{S_{2}}\right) \chi_{B S_{1}}+\bar{\phi}_{S_{2}} /\left(\bar{\phi}_{S_{1}}+\bar{\phi}_{S_{2}}\right) \chi_{B S_{2}}$, respectively. In particular, when the volume fraction ratio of $S_{1}$ and $S_{2}\left(\bar{\phi}_{S_{1}} / \bar{\phi}_{S_{2}}\right)$ is fixed at a value $\geqslant 7 / 3$ such that the effective parameters $0.4 \leqslant \chi_{A S} \leqslant 0.46$ and $\chi_{B S}=0.4$, a transition from $L \rightarrow D$ occurs as $\bar{\phi}_{C}$ decreases, similar to the block copolymer in the presence of only one neutral $S_{1}$ solvent. As $\bar{\phi}_{S_{1}} / \bar{\phi}_{S_{2}}$ falls below $7 / 3\left(0.46 \leqslant \chi_{A S} \leqslant 0.6\right.$ and $\left.\chi_{B S}=0.4\right)$, the preferentiality of the solvent for the $B$-block becomes significant enough so that a 

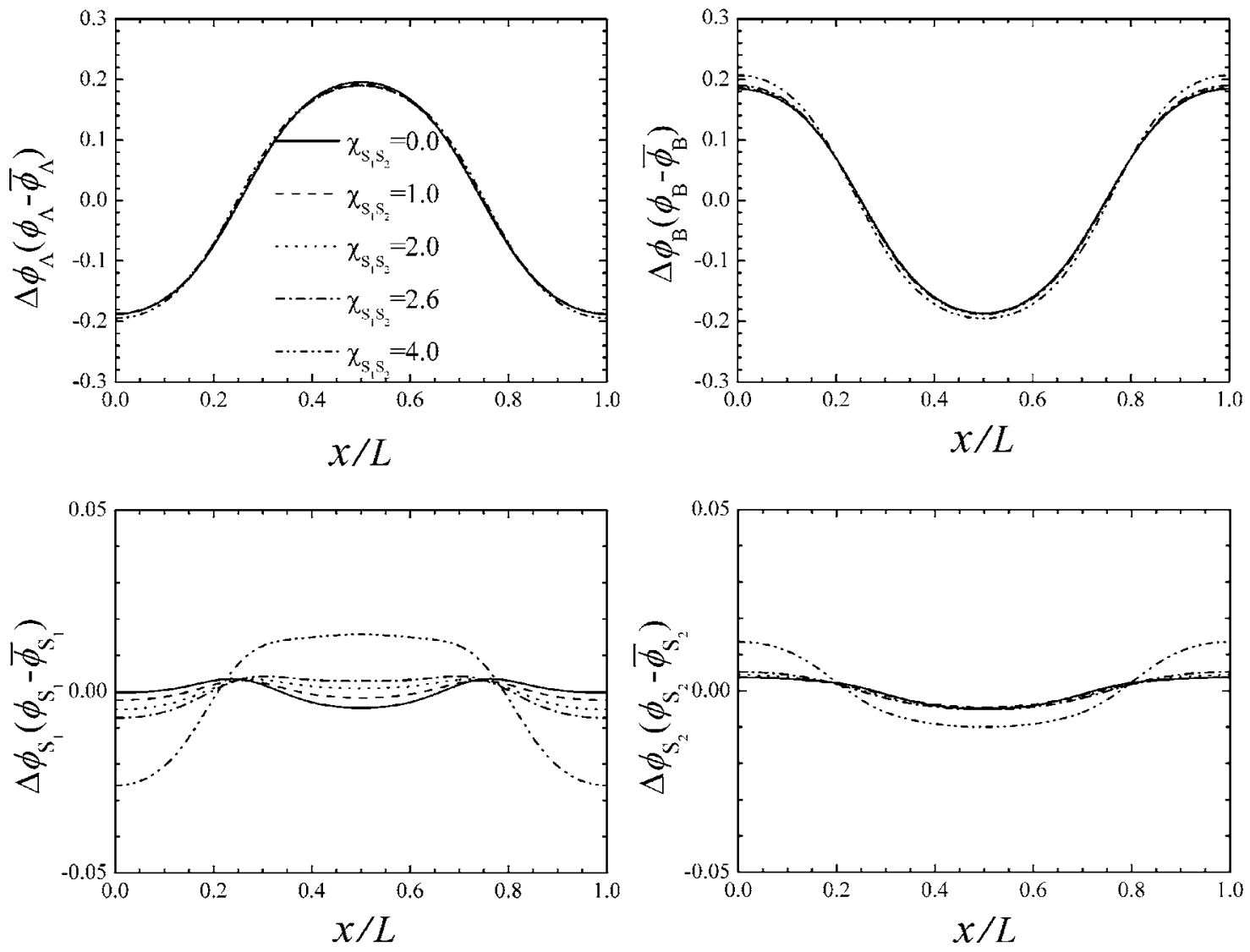

FIG. 4. Deviation of volume fraction profiles of component $A, B, S_{1}$, and $S_{2}$ within the lamellar phase for $f=0.5, N=200, \chi_{A B} N=25$, $\bar{\phi}_{C}=0.5, \bar{\phi}_{S_{1}} / \bar{\phi}_{S_{2}}=8 / 2, \chi_{A S_{1}}=\chi_{B S_{1}}=0.4, \chi_{A S_{2}}=0.6$, and $\chi_{B S_{2}}=0.4$ at various values of $\chi_{S_{1} S_{2}}$.

similar transition to the diblock copolymers in the $S_{2}$ solvent, $L \rightarrow C_{A} \rightarrow S_{A}^{\mathrm{BCC}} \rightarrow D$ is observed with decreasing $\bar{\phi}_{C}$. As $\chi_{S_{1} S_{2}}$ increases to 2.0, we obtain a similar phase diagram shown in Fig. 2(b). Though the ordered regime remains somewhat the same as that for $\chi_{S_{1} S_{2}}=0$, a slight expansion of the stable $\mathrm{L}$ phase region compresses the regime of $C_{A}$ and $S_{A}^{\mathrm{BCC}}$. This is not surprising due to the fact that a slight degree of immiscibility between $S_{1}$ and $S_{2}$ impels the neutral solvent $S_{1}$ to move forward into the $A$-rich domains, and $S_{1}$ thus behaves as a slightly $A$-selective solvent. As a result, a system initially in the stable $C_{A}$ region when $\chi_{S_{1} S_{2}}=0$ is expected to form a lamellar phase with increasing $\chi_{S_{1} S_{2}}$.

As $\chi_{S_{1} S_{2}}>2.0$, the significant immiscibility between solvents $S_{1}$ and $S_{2}$ not only drives a macrophase separation from $D \rightarrow$ two-phase $A B / S_{1}$ - and $S_{2}$-rich but also a transition from $D \rightarrow$ one ordered microstructure at lower values of $\bar{\phi}_{C}$, as presented in Fig. 2(c) where $\chi_{S_{1} S_{2}}=2.5$. This is reasonable since increasing $\chi_{S_{1} S_{2}}$ enhances the preferentiality of $S_{1}$ for $A$ and $S_{2}$ for $B$. The addition of more $S_{1}$ and $S_{2}$ to the $A B$, diblock copolymers is no longer a diluent effect, but instead, enhances the effective segregation between $A$ and $B$. Hence, ordered microphase separation is expected at lower $\bar{\phi}_{C}$. In addition, the ordered microphase region, in which $\bar{\phi}_{C}$ is higher, is enlarged. As such, by varying the ratio of solvent amount $\bar{\phi}_{S_{1}} / \bar{\phi}_{S_{2}}$, we obtain various phase transition behaviors with $\bar{\phi}_{C}$. For example, systems in which the amount of
$S_{1}\left(S_{2}\right)$ is much larger than that of $S_{2}\left(S_{1}\right)$ undergo a sequence $L\left(\rightarrow C_{A} \rightarrow \mathrm{S}_{A}^{\mathrm{BCC}}\right) \rightarrow D$ as $\bar{\phi}_{C}$ decreases when $\chi_{S_{1} S_{2}}=2.5$ [Fig. 2(c)]. When the $S_{1}$ and $S_{2}$ amounts are comparable, a sequence $L\left(\rightarrow C_{A} \rightarrow S_{A}^{\mathrm{BCC}}\right) \rightarrow D \rightarrow 1$ ordered $(O)$ phase $\left(S_{A}^{\mathrm{BCC}}\right.$ $\left.\rightarrow C_{A} \rightarrow L\right) \rightarrow 2$-phase $\left(A B / S_{1}\right.$ - and $S_{2}$-rich) is expected with decreasing $\bar{\phi}_{C}$. The tie-lines in Fig. 2(c) give the equilibrium volume fractions inside the two-phase regions as one phase rich in the $A B$ diblock and $S_{1}$ and the other phase rich in $S_{2}$. Note that we did not obtain the exact transition details associated with both microphase and macrophase separations. This difficulty occurs due to the fact that we employ a canonical ensemble approach of the SCMF theory. That is, the exact boundary between one ordered $(O)$ phase and twophase $[1(O)+1(D)$ or $2(D)]$ remains undetermined. We only locate the two-phase regime according to the Flory-Huggins thermodynamic analysis. As $\chi_{S_{1} S_{2}}$ increases, in addition to the continuous expansion of this two-phase regime at lower values of $\bar{\phi}_{C}$, we also observe that both the upper and lower ordered regions keep expanding and eventually overlap. For example, when $\chi_{S_{1} S_{2}}=2.55$ [in Fig. $2(\mathrm{~d})$ ], the stable $C_{A}$ in the upper and the lower regimes overlap leading to a single interior $C_{A}$, which also occurs with $S_{A}^{\mathrm{BCC}}$. As $\chi_{S_{1} S_{2}}$ increases to 2.6 [in Fig. 2(e)], a similar overlapping behavior for the $L$ phase is observed, which enables the stable $C_{A}$ as well as the $S_{A}^{\mathrm{BCC}}$ to move toward the right-side of the phase triangle. When $\chi_{S_{1} S_{2}}>2.6$, both the ordered microstructure and the two-phase regions continue to enlarge, as expected. 

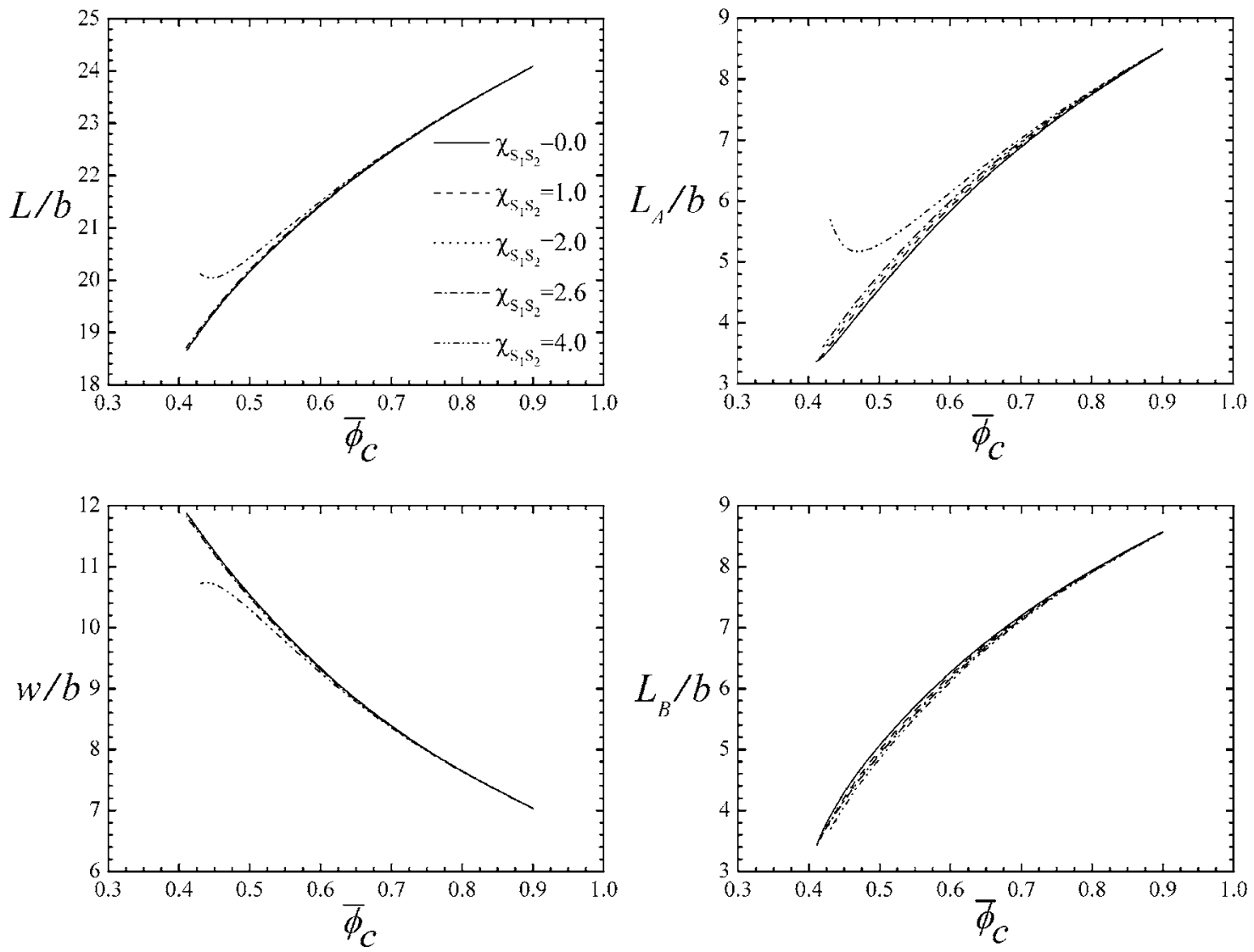

FIG. 5. Plot of lattice spacing $L, A$-rich domain width $L_{A}$, interfacial width $w$, and $B$-rich domain width $L_{B}$ vs $\bar{\phi}_{C}$ at various values of $\chi_{S_{1} S_{2}}$ for $f=0.5, N=200, \chi_{A B} N=25, \bar{\phi}_{S_{1}} / \bar{\phi}_{S_{2}}=8 / 2, \chi_{A S_{1}}=\chi_{B S_{1}}=0.4, \chi_{A S_{2}}=0.6$, and $\chi_{B S_{2}}=0.4$.

We then examine the effects of copolymer volume fraction $\bar{\phi}_{C}$, the solvent ratio $\bar{\phi}_{S_{1}} / \bar{\phi}_{S_{2}}$, and the immiscibility parameter between two solvents $\chi_{S_{1} S_{2}}$, on the distribution of each component. Figure 3 demonstrates the deviation of volume fraction profiles $\left(\phi_{I}(x)-\bar{\phi}_{I}\right)$ of component $A, B, S_{1}$, and $S_{2}$ at a series of $\chi_{S_{1} S_{2}}$ within the $L$ phase for $\bar{\phi}_{C}=0.8$ and $\bar{\phi}_{S_{1}} / \bar{\phi}_{S_{2}}=8 / 2$. As expected, the $B$-selective solvent $S_{2}$ distributes more in the $B$-rich domains than in the $A$-rich domains, regardless of the $\chi_{S_{1} S_{2}}$ value. However, though the solvent $S_{1}$ is neutral and good to both $A$ and $B$, its distribution along the $L$ phase is greatly influenced by the presence of a $B$-selective solvent $S_{2}$ due to the degree of incompatibility between these two solvents. When $\chi_{S_{1} S_{2}}=0, S_{1}$ acts as a $S_{2}$-like solvent, distributing more in the $B$-rich domains than in the $A$-rich domains. With increasing $\chi_{S_{1} S_{2}}$, more $S_{1}$ migrates toward the $A$-rich regimes in order to reduce the contacts between $S_{1}$ and $S_{2}$. $S_{1}$ thus acts more selectively for $A$ blocks. However, as there exist only a small amount of solvents, this increasing degree of solvent selectivity is slight, and solvent $S_{1}$ accumulates at the interfaces. As the copolymer volume fraction $\bar{\phi}_{C}$ decreases, a more pronounced solvent preferentiality at the same degree of $\chi_{S_{1} S_{2}}$ is expected. For example, in Fig. 4 we plot the deviation profiles for the same series of $\chi_{S_{1} S_{2}}$ but $\bar{\phi}_{C}$ decreases to $0.5\left(\bar{\phi}_{S_{1}} / \bar{\phi}_{S_{2}}\right.$ is still fixed at 8/2). Clearly, this $S_{1}$ accumulation behavior at the interfaces is not as significant as that observed in Fig. 3 at $\bar{\phi}_{C}=0.8$. Furthermore, the solvent deviation profiles at $\chi_{S_{1} S_{2}}$ $=4.0$ in Fig. 4 reveal the strong selectivity of $S_{1}$ for $A$ and $S_{2}$ for $B$. In general, we find that the solvent preferentiality increases with increasing $\chi_{S_{1} S_{2}}$ and/or decreasing $\bar{\phi}_{C}$.

Varying $\chi_{S_{1} S_{2}}$ and $\bar{\phi}_{C}$ has a great effect not only on solvent selectivity and the distribution of each component, but also on the microstructural length scales. For example, Fig. 5 shows the variation in lamellar domain spacing $L$, the width of the A-rich domains $L_{A}$, the interfacial width $w$, and the width of the $B$-rich regions $L_{B}$, with the changes in $\bar{\phi}_{C}$ for a series of $\chi_{S_{1} S_{2}}$ at a fixed ratio $\bar{\phi}_{S_{1}} / \bar{\phi}_{S_{2}}=8 / 2$. We find that the microstructural length results are almost independent of $\chi_{S_{1} S_{2}}$ until $\chi_{S_{1} S_{2}}>2.6$. This is due to the fact that lower values of $\chi_{S_{1} S_{2}}$ affect the solvent selectivity for each block slightly; the variation of the microstructural lengths with $\bar{\phi}_{C}$ is similar to that observed for a diblock copolymer in the presence of a slightly selective solvent. As expected, decreasing $\bar{\phi}_{C}$ is simply a consequence of the decreased segregation, which in turn reduces the chain stretching degree normal to the interfaces. Therefore, both domain lengths $L_{A}$ and $L_{B}$ show a decreasing behavior, and the interfacial width $w$ increases as $\bar{\phi}_{C}$ decreases. As a result, the domain spacing $L$ decreases as $\bar{\phi}_{C}$ decreases. At the same time, as $\chi_{S_{1} S_{2}}$ increases to $\chi_{S_{1} S_{2}}$ $=4.0$, we observe that both $L_{A}$ and $L$ first decrease with $\bar{\phi}_{C}$ 

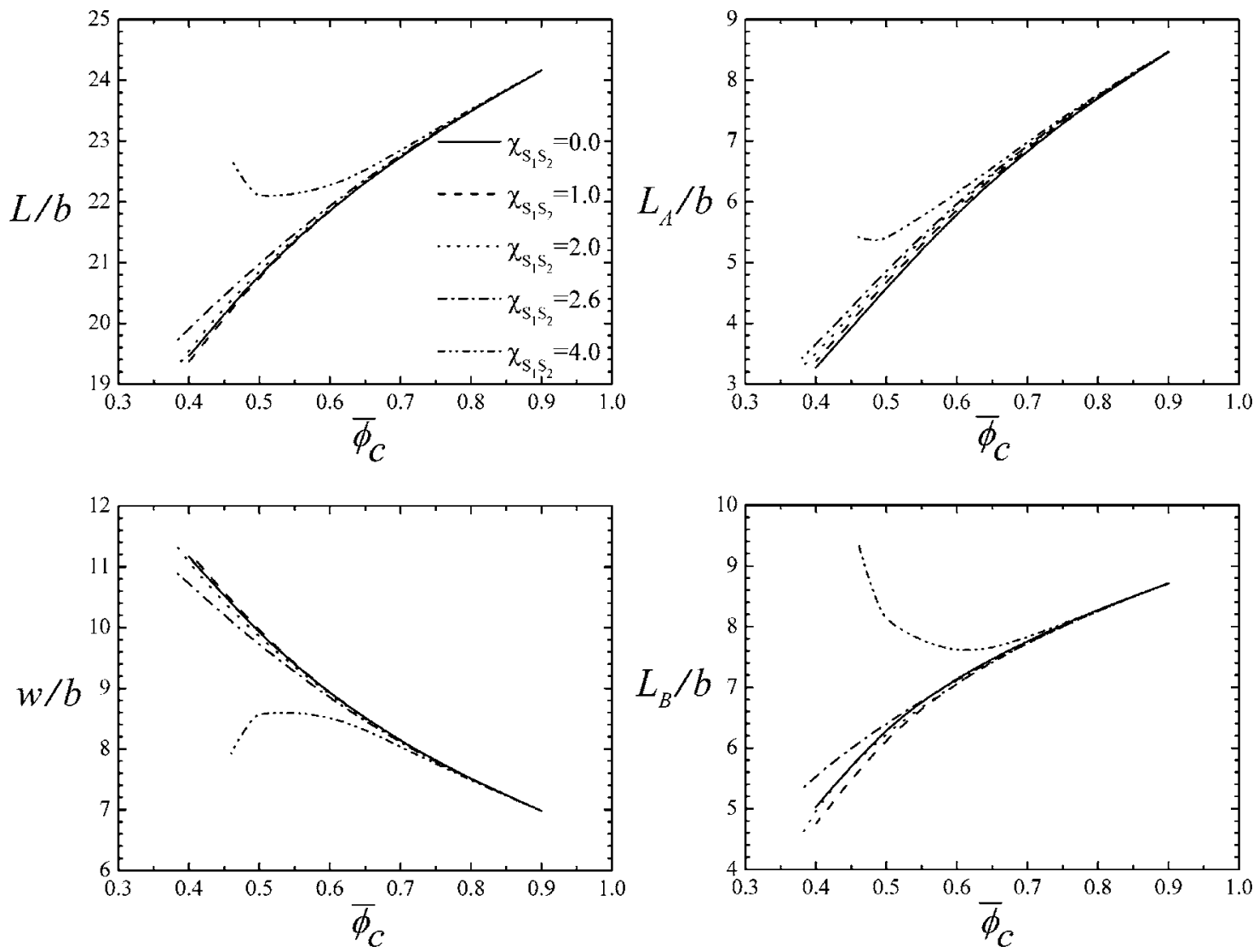

FIG. 6. Plot of lattice spacing $L, A$-rich domain width $L_{A}$, interfacial width $w$, and $B$-rich domain width $L_{B}$ vs $\bar{\phi}_{C}$ at various values of $\chi_{S_{1} S_{2}}$ for $f=0.5, N=200, \chi_{A B} N=25, \bar{\phi}_{S_{1}} / \bar{\phi}_{S_{2}}=2 / 8, \chi_{A S_{1}}=\chi_{B S_{1}}=0.4, \chi_{A S_{2}}=0.6$, and $\chi_{B S_{2}}=0.4$.

decreasing and then increase significantly as $\bar{\phi}_{C}$ decreases further. This increasing behavior at lower values of $\bar{\phi}_{C}$ is mainly attributed to the strong selectivity of $S_{1}$ to $A$ and $S_{2}$ to $B$. Because of the very small amounts of solvent $S_{2}$, its segregation to the $B$-rich domains is not significant enough to enlarge the $B$-rich domains. As the ratio of $S_{2}$ in the solvents increases, the increase of $L_{B}$ becomes more significant. For example, Fig. 6 plots the variation of $L, L_{A}$, $w$, and $L_{B}$, with $\bar{\phi}_{C}$ for the same series of $\chi_{S_{1} S_{2}}$ but $\bar{\phi}_{S_{1}} / \bar{\phi}_{S_{2}}$ changes to $2 / 8$; the increasing behavior of $L$ with increasing $\chi_{S_{1} S_{2}}$ at lower values of $\bar{\phi}_{C}$ is mainly due to the great increase of $L_{B}$, although the interfacial width $w$ is reduced. To manifest the effects of solvent ratio on the microstructural lengths at various degrees of solvent immiscibility $\chi_{S_{1} S_{2}}$, we present the corresponding $L, L_{A}, w$, and $L_{B}$, as a function of the percentage of $S_{2}$ in the solvents at a series of $\chi_{S_{1} S_{2}}$ when $\bar{\phi}_{C}$ is fixed at 0.5 in Fig. 7. It is clear that regardless of the solvent ratio, $L_{A}$ shows an increasing behavior with $\chi_{S_{1} S_{2}}$. However, $L_{B}$ increases significantly only when the immiscibility $\chi_{S_{1} S_{2}}$ reaches at least 4.0. Moreover, we observe that the increasing degree of the overall domain spacing $L$ at $\chi_{S_{1} S_{2}}=4.0$ becomes most significant when the volume fractions of $S_{1}$ and $S_{2}$ are comparable. This is due to the fact that both $L_{A}$ and $L_{B}$ are also enlarged, primarily by the strong segregation of $S_{1}$ to $A$-rich and $S_{2}$ to $B$-rich domains.

\section{CONCLUSIONS}

We study the phase behavior as well as the microstructural domain sizes for an $A B$ diblock copolymer in the presence of a neutral solvent $S_{1}$ and a $B$-selective solvent $S_{2}$, by self-consistent mean-field (SCMF) theory. As in the diblock copolymer solutions when only one solvent is added, the possible formed phases include $L$, normal and inverted $G$, $(P L)$, normal and inverted $C$, and normal and inverted $S^{\mathrm{BCC}} / S^{\mathrm{FCC}}$. Though the addition of a second solvent cannot induce new morphology types, we find that both the phase behavior and the microstructural sizes are strongly affected by the immiscibility parameter between two solvents $\chi_{S_{1} S_{2}}$, the solvent ratio, and the copolymer volume fraction.

With increasing $\chi_{S_{1} S_{2}}$, we observe that the ordered microphase region, in which $\bar{\phi}_{C}$ is higher and thus the incompatibility between $A / B \chi_{A B}$ drives the microphase separation, is expanded. Furthermore, when $\chi_{S_{1} S_{2}}>2.0$, due to the fact that the two solvents are significantly incompatible, the oneordered phase regimes as well as the two-macrophase $A B / S_{1}$ - and $S_{2}$-rich regimes are induced in the systems at lower values of $\bar{\phi}_{C}$. It is interesting to find that these two ordered regimes keep expanding and eventually overlap with a further increase in $\chi_{S_{1} S_{2}}$. These results are not surprising since increasing $\chi_{S_{1} S_{2}}$ is analogous to enhancing the preferentiality of $S_{1}$ for $A$ and $S_{2}$ for $B$ and thereafter the effective segregation between $A$ and $B$. 

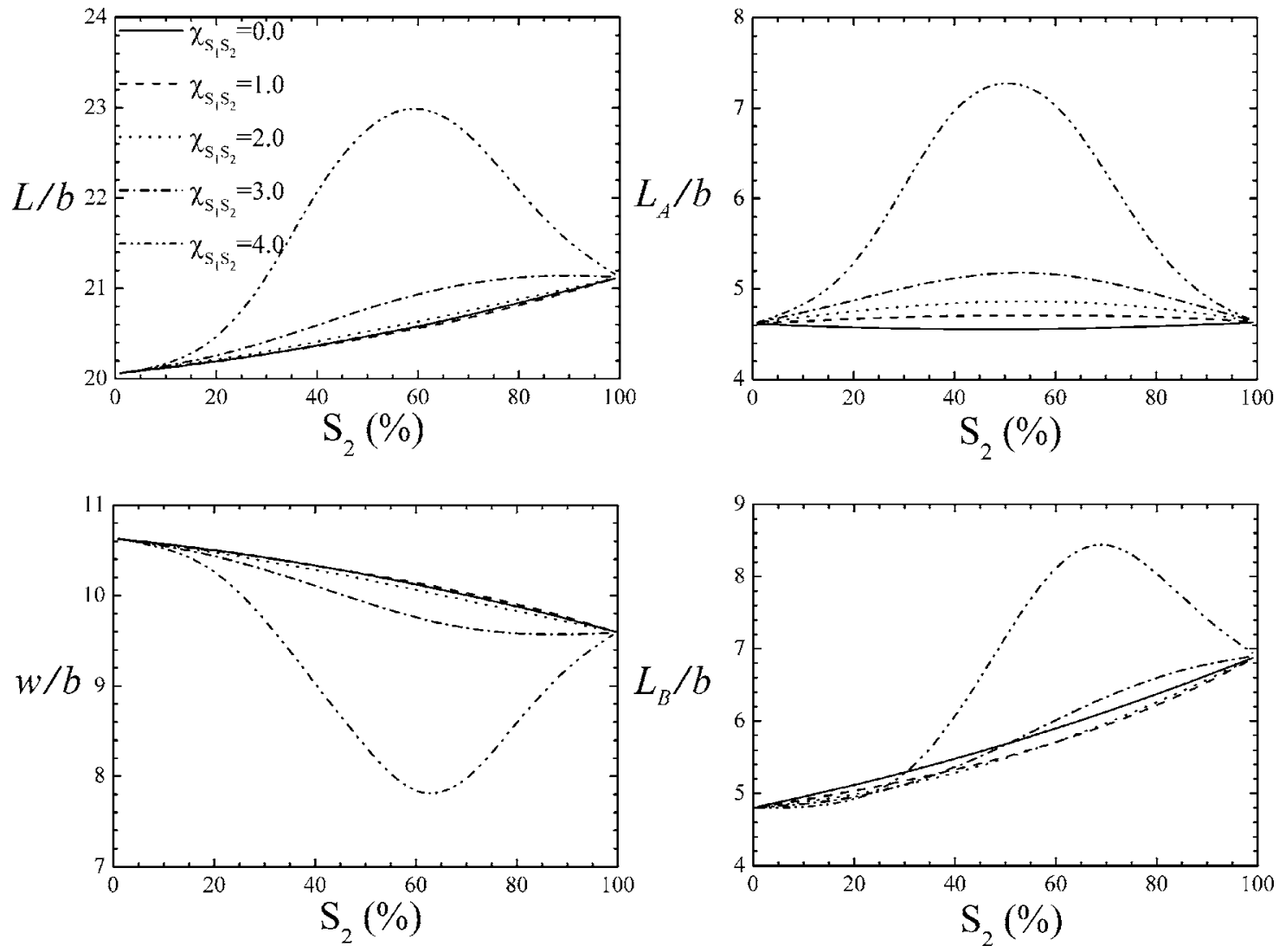

FIG. 7. Variation of lattice spacing $L, A$-rich domain width $L_{A}$, interfacial width $w$, and $B$-rich domain width $L_{B}$ with the percentage of $S_{2}$ in the solvents and $\chi_{S_{1} S_{2}}$ for $f=0.5, N=200, \chi_{A B} N=25, \bar{\phi}_{C}=0.5, \chi_{A S_{1}}=\chi_{B S_{1}}=0.4, \chi_{A S_{2}}=0.6$, and $\chi_{B S_{2}}=0.4$.

In the analysis of the lamellar domain lengths, when $\chi_{S_{1} S_{2}}$ is not significant, the domain spacing $L$ decreases as $\bar{\phi}_{C}$ decreases. This trend is similar to that observed for a diblock copolymer in the presence of a slightly selective solvent. While at larger values of $\chi_{S_{1} S_{2}}$, the domain spacing first shows a decreasing trend with decreasing $\bar{\phi}_{C}$ and then an increasing behavior with a further decrease in $\bar{\phi}_{C}$, as in the diblock copolymer solutions when a strongly selective solvent is added. This domain spacing increasing behavior is not surprising since when more immiscible solvents are added (i.e., decreasing $\bar{\phi}_{C}$ ), more $S_{1}$ and $S_{2}$ swell the $A$-rich domains and the $B$-rich domains, respectively. In further, this swelling behavior is the most significant when the amounts of these two solvents are comparable. As in the diblock copolymer solutions when only one solvent is added, we believe that these structural length results analyzed in the lamellar phase also hold true in other structures.

\section{ACKNOWLEDGMENT}

This work was supported by the National Science Council of the Republic of China through Grant No. NSC 94-2216E-002-027.
[1] N. Garti, A. Spernath, A. Aserin, and R. Lutz, Soft Matter 1, 206 (2005)

[2] T. P. Lodge, Macromol. Chem. Phys. 204, 265 (2003).

[3] N. Hadjichristidis, S. Pispas, and G. Floudas, Block Copolymers: Synthetic Strategies, Physical Properties, and Applications (Wiley, New Jersey, 2003).

[4] Self-Assembly, edited by B. H. Robinson (IOS Press, Amsterdam, 2003).

[5] I. W. Hamley, The Physics of Block Copolymers (Oxford University Press, New York, 1998).

[6] P. Alexandridis, U. Olsson, and B. Lindman, Macromolecules
28, 7700 (1995).

[7] P. Alexandridis, D. Zhou, and A. Khan, Langmuir 12, 2690 (1996).

[8] P. Holmqvist, P. Alexandridis, and B. Lindman, Macromolecules 30, 6788 (1997).

[9] P. Alexandridis, U. Olsson, and B. Lindman, Langmuir 14, 2627 (1998).

[10] B. Svensson, P. Alexandridis, and U. Olsson, J. Phys. Chem. B 102, 7541 (1998).

[11] B. Svensson, P. Alexandridis, and P. Linse, Macromolecules 32, 5435 (1999). 
[12] P. Kipkemboi, A. Khan, B. Lindman, and V. Alfredsson, Can. J. Chem. 81, 897 (2003).

[13] R. G. Larson, J. Phys. France II 6, 1441 (1996).

[14] A. D. Mackie, O. Kaan, and A. Z. Panagiotopoulos, J. Chem. Phys. 104, 3718 (1996).

[15] S. K. Talsania, L. A. Rodriguez-Guadarrama, K. K. Mohanty, and R. Rajagopalan, Langmuir 14, 2684 (1998).

[16] S. Y. Kim, A. Z. Panagiotopoulos, and M. A. Floriano, Mol. Phys. 100, 2213 (2002).

[17] L. Rekvig, M. Kranenburg, J. Vreede, B. Hafskjold, and B. Smit, Langmuir 19, 8195 (2003).

[18] S. G. Schulz, H. Kuhn, G. Schmid, C. Mund, and J. Venzmer, Colloid Polym. Sci. 283, 284 (2004).

[19] A. C. Shi, in Developments in Block Copolymer Science and Technology, edited by I. W. Hamley (Wiley, New York, 2004).

[20] M. W. Matsen and M. Schick, Phys. Rev. Lett. 72, 2660 (1994); M. W. Matsen and F. S. Bates, Macromolecules 29, 1091 (1996).

[21] K. M. Hong and J. Noolandi, Macromolecules 16, 1083 (1983).

[22] M. D. Whitmore and J. Noolandi, J. Chem. Phys. 93, 2946 (1990).

[23] M. D. Whitmore and J. D. Vavasour, Macromolecules 25, 2041 (1992).

[24] C. I. Huang and T. P. Lodge, Macromolecules 31, 3556 (1998).

[25] E. Helfand and Y. Tagami, J. Chem. Phys. 56, 3592 (1972).

[26] K. J. Hanley and T. P. Lodge, J. Polym. Sci., Part B: Polym. Phys. 36, 3101 (1998).

[27] K. J. Hanley, T. P. Lodge, and C. I. Huang, Macromolecules
33, 5918 (2000).

[28] T. P. Lodge, K. J. Hanley, B. Pudol, and V. Alahapperuma, Macromolecules 36, 816 (2003).

[29] T. Hashimoto, M. Shibayama, and H. Kawai, Macromolecules 16, 1093 (1983).

[30] M. Shibayama, T. Hashimoto, H. Hasegawa, and H. Kawai, Macromolecules 16, 1427 (1983).

[31] T. P. Lodge, C. Pan, X. Jin, Z. Liu, J. Zhao, W. W. Maurer, and F. S. Bates, J. Polym. Sci., Part B: Polym. Phys. 33, 2289 (1995).

[32] M. Banaszak and M. D. Whitmore, Macromolecules 25, 3406 (1992).

[33] J. Noolandi, A. C. Shi, and P. Linse, Macromolecules 29, 5907 (1996).

[34] K. Zhang and A. Khan, Macromolecules 28, 3807 (1995).

[35] C. I. Huang and H. Y. Hsueh, Polymer 47, 6843 (2006).

[36] M. Shibayama, T. Hashimoto, and H. Kawai, Macromolecules 16, 16 (1983).

[37] G. A. McConnell and A. P. Gast, Macromolecules 30, 435 (1997).

[38] T. P. Lodge, B. Pudil, and K. J. Hanley, Macromolecules 35, 4707 (2002).

[39] J. Bang and T. P. Lodge, J. Phys. Chem. B 107, 12071 (2003).

[40] C. Lai, W. B. Russel, and R. A. Register, Macromolecules 35, 841 (2002); 35, 4044 (2002).

[41] International Tables for X-ray Crystallography, edited by N. F. M. Henry and K. Lonsdale (Kynoch, Birmingham, 1969).

[42] C. Huang, M. Olvera de la Cruz, and B. W. Swift, Macromolecules 28, 7996 (1995). 